\title{
Detection of Keplerian dynamics in a disk around the post-AGB star AC Herculis
}

\author{
V. Bujarrabal ${ }^{1}$, A. Castro-Carrizo ${ }^{2}$, J. Alcolea ${ }^{3}$, and H. Van Winckel ${ }^{4}$ \\ 1 Observatorio Astronómico Nacional (OAN-IGN), Apartado 112, 28803 Alcalá de Henares, Spain \\ e-mail: v.bujarrabal@oan.es \\ ${ }^{2}$ Institut de Radioastronomie Millimétrique, 300 rue de la Piscine, 38406 Saint-Martin-d'Hères, France \\ 3 Observatorio Astronómico Nacional (OAN-IGN), C/ Alfonso XII, 3, 28014 Madrid, Spain \\ ${ }^{4}$ Instituut voor Sterrenkunde, K.U. Leuven, Celestijnenlaan 200B, 3001 Leuven, Belgium
}

Received 26 January 2015 / Accepted 4 February 2015

\section{ABSTRACT}

\begin{abstract}
Aims. So far, only one rotating disk has been clearly identified and studied in AGB or post-AGB objects (in the Red Rectangle), by means of observations with high spectral and spatial resolution. However, disks are thought to play a key role in the late stellar evolution and are suspected to surround many evolved stars. We aim to extend our knowledge on these structures.

Methods. We present interferometric observations of ${ }^{12} \mathrm{CO} J=2-1$ emission from the nebula surrounding the post-AGB star AC Her, a source belonging to a class of objects that share properties with the Red Rectangle and show hints of Keplerian disks.

Results. We clearly detect the Keplerian dynamics of a second disk orbiting an evolved star. Its main properties (size, temperature, central mass) are derived from direct interpretation of the data and model fitting. With this we confirm that there are disks orbiting the stars of this relatively wide class of post-AGB objects.
\end{abstract}

Key words. stars: AGB and post-AGB - circumstellar matter - radio lines: stars - planetary nebulae: individual: AC Her

\section{Introduction}

Disks orbiting asymptotic giant branch (AGB) and post-AGB stars are thought to play a major role in the late AGB evolution and planetary nebulae formation. In the vast majority of cases, the circumstellar envelopes around AGB stars are roughly spherical and in isotropic expansion at moderate velocities (10-20 $\mathrm{km} \mathrm{s}^{-1}$ ), see, for example, Castro-Carrizo et al. (2010). Most protoplanetary and planetary nebulae (PPNe, PNe) show a clear axial symmetry and fast bipolar outflows $\left(\sim 100 \mathrm{~km} \mathrm{~s}^{-1}\right)$, which carry a good fraction of the total mass $\left(\sim 0.1 M_{\odot}\right)$ and very large amounts of linear momentum, see, for instance, Bujarrabal et al. (2001) and Alcolea et al. (2001). This spectacular transition takes place in a very short time, a thousand or a few thousand years. The most widely accepted scenario to theoretically explain this evolutionary phase implies that material is reaccreted by the star or a companion from a rotating disk, followed by the launching of very fast jets, in a process similar to that at work in forming stars, see Soker (2002), Blackman \& Lucchini (2014), and references therein.

Such orbiting disks have been frequently searched for, but their detection has been extremely difficult. Except for one source (the Red Rectangle, see below), in all AGB shells or post-AGB nebulae in which spectroscopical observations allow measuring the velocity field, only expansion has been detected. Many of them show oblate, even very flat structures, but without any sign of rotation in the observations. This is the case of axisymmetric shells around semiregular variables (e.g., Libert et al. 2010; Castro-Carrizo et al. 2010) and of flat equatorial structures that are often found in bipolar PPNe (e.g. Alcolea et al. 2007; Castro-Carrizo et al. 2002, 2012).

Various indications of the existence of Keplerian disks have been proposed. The very low expansion velocity $\left(\$ 1 \mathrm{~km} \mathrm{~s}^{-1}\right)$ sometimes found in the equatorial structures mentioned above suggests that the gas is ejected from a stable, and therefore probably Keplerian, component at long distances to the star, in which outward forces are weak. On the other hand, a class of optically bright post-AGB stars are found to show a remarkable near-infrared excess, which has been attributed to the emission of hot dust at about $1000 \mathrm{~K}$. The high temperature would show that dust grains are kept close to the star, again in a stable disk structure, see Van Winckel (2003) and van Aarle et al. (2011). These stars are found to be systematically multiple (Van Winckel et al. 2009) and the grains show a high degree of evolution (i.e., a high critallinity; Gielen et al. 2011), which supports this interpretation.

Systematic observations of the ${ }^{12} \mathrm{CO}$ and ${ }^{13} \mathrm{CO} J=2-1$ and $J=1-0$ lines in objects with such a near-infrared excess (Bujarrabal et al. 2013a) have shown narrow line profiles formed by a single or double peak and weaker wings, which are very similar to those found in the Red Rectangle. These profiles are also very similar to those in young stars known to be surrounded by remnants of IS material in rotation and to predictions of models of CO emission from orbiting disks, see Guilloteau \& Dutrey (1998), Guilloteau et al. (2013), Bujarrabal \& Alcolea (2013), etc. These results led Bujarrabal et al. (2013a) to conclude that the $\mathrm{CO}$ lines in these sources probably come from Keplerian disks.

However, the unambiguous detection of the Keplerian dynamics requires very accurate spectroscopic observations with high spatial and spectral resolution, able to describe the velocity field at subarcsec scales and with spectral resolutions better than $0.5 \mathrm{~km} \mathrm{~s}^{-1}$. To date, this type of observations has resulted in the detection of rotation in just one evolved object, the Red Rectangle, a well known PPN showing the abovementioned near-infrared excess (Bujarrabal et al. 2005, 2013b). 


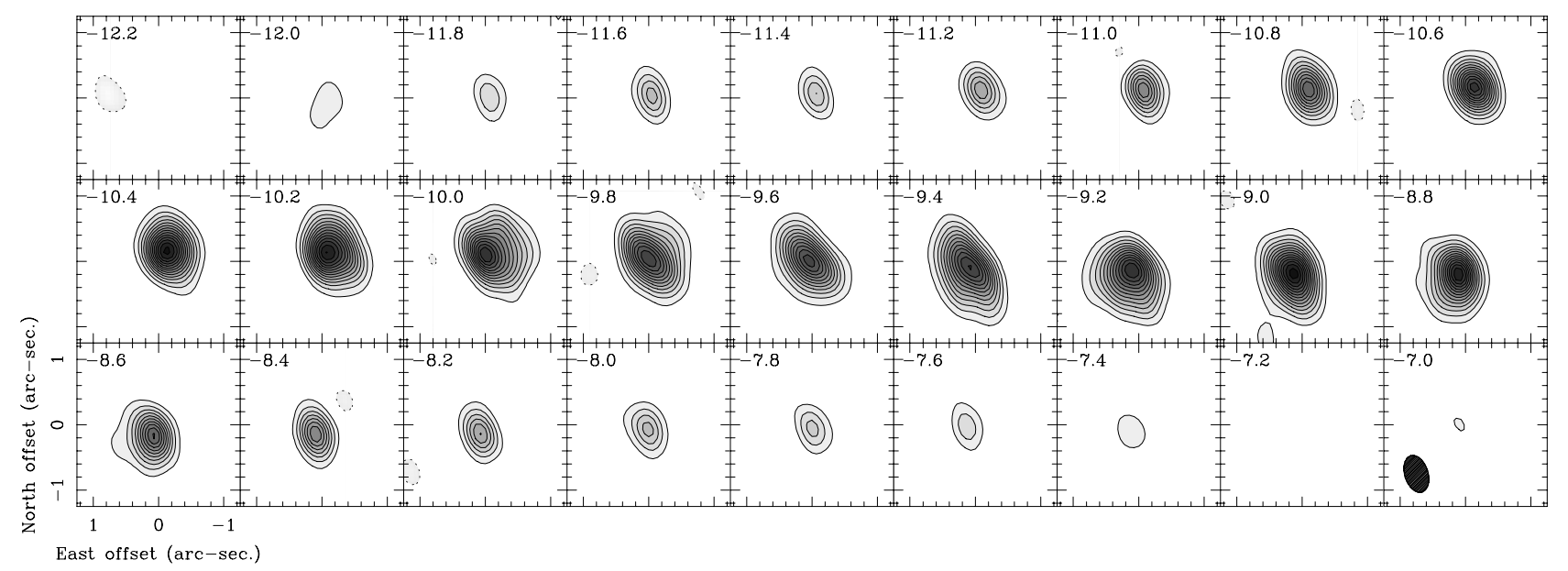

Fig. 1. PdBI maps per velocity channel of ${ }^{12} \mathrm{CO} J=2-1$ emission from AC Her. The LSR velocity is indicated in the upper left corners. The first contour and step are $16 \mathrm{mJy}$, negative contours are indicated by dashed lines. The dark ellipse in the last panel shows the half-power extent of the synthetic beam. The conversion factor from Jy to K is $110 \mathrm{~K}$ per Jy/beam, the rms noise measured in the maps is about $4 \mathrm{mJy} / \mathrm{beam}$.

Radiointerferometric observations of CO rotational lines undoubtedly showed a rotating equatorial disk, together with gas in slow expansion that seems to be extracted from the disk. The main properties of the nebula (extent, structure, dynamics) and gas physical conditions (temperature, density) could be studied.

Several attempts to observe similar objects in this way have been carried out (particularly deep in 89 Her and IRAS 19125+0343; Bujarrabal et al. 2007), but only expanding gas was found. The detection of additional cases of rotating disks around post-AGB stars is necessary to confirm our interpretation of low-resolution CO data and that Keplerian disks are relatively common among evolved nebulae. It is also necessary to study the main properties of disks in a wide class of sources.

We present here the second firm detection of a Keplerian disk around a post-AGB star, AC Her. AC Her, a binary star showing RV Tau variability, is placed at a distance $D \sim 1.6 \mathrm{kpc}$, with a total luminosity $\sim 2500 L_{\odot}$ (Hillen et al. 2015). Its CO profiles are narrow (Bujarrabal et al. 2013a) and were attributed to rotating structures. From optical and interferometric IR data, Hillen et al. deduced the presence of a dust disk around AC Her with an inclination with respect to the line of sight of about $45^{\circ}$.

\section{Observations}

Observations of ${ }^{12} \mathrm{CO} J=2-1$ line emission $(230.538 \mathrm{GHz})$ in AC Her were performed with the Plateau de Bure interferometer (PdBI; project XB74). Data were obtained with the $6 \mathrm{~Bq}$ and 6Aq array configurations in March 2014. After a first calibration based on observations of nearby quasars, data were selfcalibrated by using the central continuum source. A total flux of $30 \mathrm{mJy}$ was obtained for the unresolved continuum by averaging a $3.6 \mathrm{GHz}$ bandwidth free of line emission in each receiver polarization. A synthetic beam of $0.58 \times 0.35$ in size (at half power, $\mathrm{PA}=17^{\circ}$ ) was obtained with data natural weights, slightly worse than expected because of limited $u v$-coverage in the extended baselines.

The CO results are presented in Fig. 1 with a channel spacing of $0.2 \mathrm{~km} \mathrm{~s}^{-1}$, but the detailed data inspection was performed with spectral resolutions of $0.1 \mathrm{~km} \mathrm{~s}^{-1}$. Note that the continuum emission was subtracted from the data shown in Fig. 1 to better identify the weak emission at extreme velocities. From comparison with single-dish profiles (Bujarrabal et al. 2013a), we deduce that $30-40 \%$ of the flux was filtered out in the interferometric maps. We hence detect the main and most compact component in $\mathrm{CO}$, which seems to be a disk whose projection is elongated in the direction $\mathrm{PA}=135^{\circ}$. The position-velocity diagram along this direction is shown in Fig. 2, where the typical Keplerian rotation pattern is conspicuous, while the plot in the perpendicular direction is completely symmetric with respect to both velocity and position, as also expected for a Keplerian disk.

\section{Results}

Some results can be directly and reliably extracted from our maps. It is necessary to deduce these robust results independently of our model fitting (Sect. 3.1), because, as we show below, such models contain many uncertain parameters.

The velocity-position diagram along PA $\sim 135^{\circ}$ is very accurately coincident with those characteristic of Keplerian dynamics (for young and evolved objects, see, e.g., Guilloteau \& Dutrey 1998; Isella et al. 2007; Bujarrabal et al. 2005, 2008). This value gives the inclination angle of the disk in the plane of the sky and cannot vary by more than $\pm 5^{\circ}$, otherwise the rotation features start to appear also in cuts along the perpendicular direction. We conclude solely from inspecting this diagram that the detected $\mathrm{CO}$ emission from AC Her comes from an orbiting disk. The non-negligible amount of flux filtered out by the interferometer (Sect. 2) must come from a relatively extended component, which could be gas in expansion, as found in the Red Rectangle and 89 Her (Sect. 1).

If we assume that the ${ }^{12} \mathrm{CO} J=2-1$ lines are opaque, which is reasonable in view of the low ${ }^{12} \mathrm{CO} /{ }^{13} \mathrm{CO}$ line ratio found in this object (Bujarrabal et al. 2013a), we can directly estimate from the measured brightness the line excitation temperature. The values so derived are a good estimate of the kinetic temperature, since low- $J$ lines are easily thermalized for the expected high densities (see, e.g., Bujarrabal \& Alcolea 2013, and Sect. 3.1). The peak brightness in our maps is $\sim 25 \mathrm{~K}$, corresponding to a kinetic temperature $\sim 30 \mathrm{~K}$ (after taking into account the conversion from brightness temperature to RayleighJeans equivalent temperature for the $J=2-1$ frequency). This is an average value within the synthetic beam; dilution can be important in the inner (hot and rapidly rotating) regions, so this value is probably more representative of the temperature in the outer disk. Bujarrabal et al. (2013a) assumed a typical temperature in the disks of $70-100 \mathrm{~K}$ from data on the Red Rectangle; 


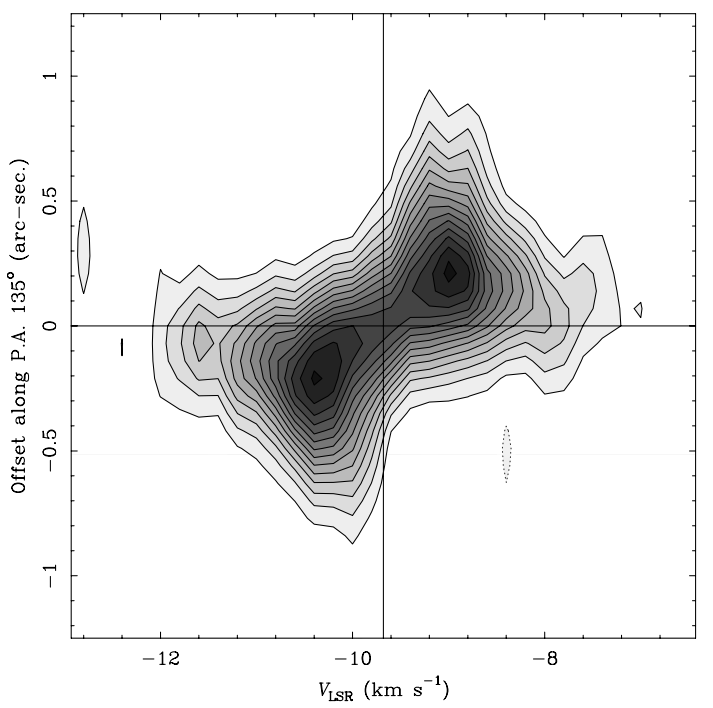

Fig. 2. Position velocity diagram along the disk direction, $\mathrm{PA}=135^{\circ}$. Color scale and contours are the same as in Fig. 1.

clearly, the disk around AC Her is significantly cooler in spite of its smaller size (see below).

The measured extent of the emitting region, $\sim 11^{\prime \prime} 5$, is significantly larger than that deduced from single-dish observations (0.'7 Bujarrabal et al. 2013a) comparing single-dish profiles with expectations for a disk with a temperature similar to that of the Red Rectangle. This discrepancy is explained by the lower temperature we find in $\mathrm{AC}$ Her. The disk around $\mathrm{AC}$ Her is much smaller than that found in the Red Rectangle (about 6" in diameter), however; in linear units, the AC Her disk is about 2.5 times smaller (the Red Rectangle is placed at $\sim 700 \mathrm{pc}$ ).

The total velocity range and separation between the brightness peaks in AC Her are much smaller than in the Red Rectangle in spite of the smaller size of the disk of AC Her. We can graphically see this effect by comparing our Fig. 2 with Fig. 3 in Bujarrabal et al. (2005) (particularly the $J=1-0$ line of the Red Rectangle, because of its lower size/resolution ratio). Provided that the disk around AC Her is not seen face-on (Sect. 1), we deduce that the mass of the stellar component must be lower than for the Red Rectangle; see model calculations in Sect. 3.1. No direct indication of expanding gas (in addition to the Keplerian disk), as that found in the Red Rectangle and $89 \mathrm{Her}$, is seen in our data. There is a weak absorption at about $-11.5 \mathrm{~km} \mathrm{~s}^{-1}$ LSR that could be due to gas in expansion, but its identification and interpretation in these terms are very tentative. We hope that more accurate observations will throw light on this question.

\subsection{Model calculations}

Our modeling of the disk orbiting AC Her is based on that we performed to explain our maps of the Red Rectangle. We used two codes. The first one solves the "exact" radiative transfer equations in two dimensions for very many transitions and the level populations for very many representative points in the disk. A disk model is assumed, including the disk structure and kinematics, as well as the distribution of the main physical parameters. See the description of the calculations and the detailed discussion of their accuracy in Bujarrabal \& Alcolea (2013). (However, our calculations show that the low- $J$ CO transitions are thermalized for typical post-AGB disk conditions, as also

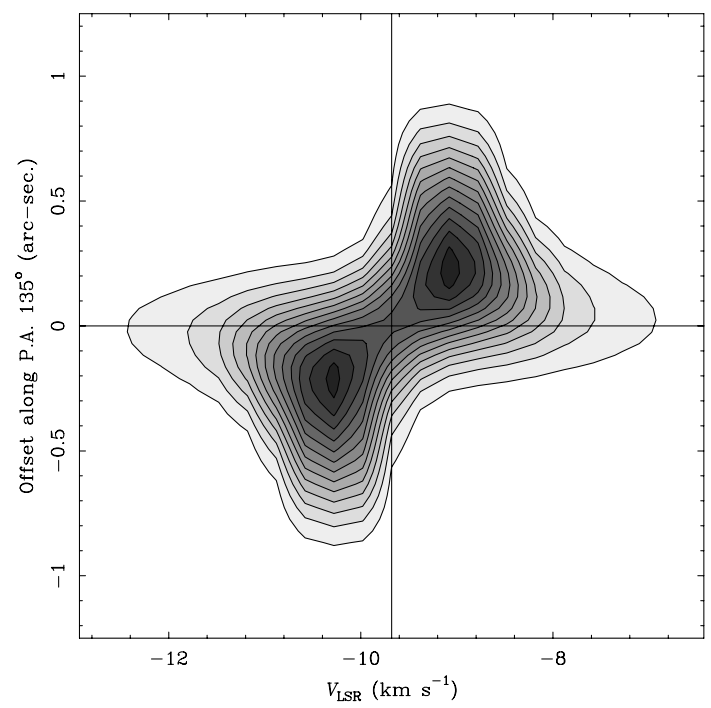

Fig. 3. Position velocity diagram predicted by our best-fit model. All units, scales, and contours are the same as in Fig. 2.

concluded in previous works; therefore, simpler excitation calculations, even assuming LTE level populations, yield similar results.) In a second step, we solved the radiative transfer equation for the lines of sight pointing at the observer, computed the predicted brightness distribution in the plane of the sky and convolved it with the interferometer synthetic beam. This led to maps and P-V cuts that can be directly compared with those observed. A similar code was used in previous papers (Bujarrabal et al. 2005, 2008, 2013b).

There are fewer data for AC Her than for the Red Rectangle. Accordingly, we kept the model as simple as possible. We took as starting point the disk structure found in the Red Rectangle disk, but scaled it down by about a factor two since the disk around AC Her is clearly smaller. We assumed no expansion, since no indication of a departure from Keplerian dynamics has been found in our maps. We assumed similar laws for the density and temperature, $n \propto 1 / r^{2}$ and $T \propto 1 / r^{0.7}$, where $r$ is the distance to the star, the proportionality constants being free parameters. The inclination of the disk (with respect to line of sight) is a free parameter, but it is assumed to range between 30 and 60 degrees (Sect. 1). We show below that all these assumptions are confirmed to be reasonable by our fitting, which leads to very satisfactory results in spite of the few free parameters.

The results of our best fitting are summarized in Fig. 3. The agreement with the observations is remarkably good and clearly confirms the Keplerian dynamics of the disk around AC Her.

Our best-fitting model is described by $n=3.5 \times$ $10^{5}\left(r_{0} / r\right)^{2} \mathrm{~cm}^{-3}, T=55\left(r_{0} / r\right)^{0.7} \mathrm{~K}, V_{\mathrm{rot}}=1.6 \sqrt{r_{0} / r} \mathrm{~km} \mathrm{~s}^{-1}$, and outer radius $R_{\text {out }}=1.7 \times 10^{16} \mathrm{~cm} . r_{0}=4.5 \times 10^{15} \mathrm{~cm}, r_{0}$ is the radius that defines the central decrease of the disk thickness (see description in our previous works) and is taken to be equal to the disk thickness (to further limit the free parameters). We took the ${ }^{12} \mathrm{CO}$ abundance to be $X\left({ }^{12} \mathrm{CO}\right)=1.5 \times 10^{-4}$, slightly smaller than that found for the Red Rectangle because of the weak emission of the source and its low ${ }^{12} \mathrm{CO} /{ }^{13} \mathrm{CO}$ line ratio (despite the weak lines); this value is practically an assumption because the low CO lines are almost thermalized in our case and the density and the molecular abundances are not independent parameters.

The inclination of the disk plane with respect to the north (PA) is assumed to be $135^{\circ}$, the disk position angle derived from our observations. We find a value of the disk inclination with respect to the line of sight (which is equal to the inclination of the 
disk axis with respect to the plane of the sky), $i \sim 45^{\circ}$, compatible with indications from other studies (Sect. 1). Our calculations indicate an uncertainty in this value of $\sim 10^{\circ}$ from comparing the predicted and observed extents in the directions of the equator and of the rotation axis: for higher angles, the predicted brightness extent in the axial direction is equal to or higher than that in the equator direction, in contrast to the observations (we recall that the angular resolution is better in the projected equator direction), while for low values of $i$ the contrast is significantly higher than observed. The sense of $i$ is not given by our data, nor do we have other information on it.

From the disk dynamics and inclination derived here, we deduce that the mass of the central stellar system is $\sim 1 M_{\odot}$. This value is somewhat lower than that derived from the binary movements in AC Her $\left(\sim 1.8 M_{\odot}\right.$, Van Winckel et al. 1998) and than the value found from the CO maps of the Red Rectangle $\left(1.7 M_{\odot}\right)$. The difference can be due to the relatively uncertain distance value, $D$, or to a slightly higher inclination, $i$, within the acceptable range mentioned above. (Our central mass estimate is roughly proportional to $D$ and $\cos ^{-2}(i)$.)

The total mass of the nebula, derived from integrating the disk densities in the model, is also low, $M_{\text {neb }} \sim 1.2 \times 10^{-3} M_{\odot}$, similar to the mass deduced for other similar objects (Bujarrabal et al. 2013a; Bujarrabal \& Alcolea 2013), but lower than the mass of the Red Rectangle disk, $\sim 6 \times 10^{-3} M_{\odot}$. It is slightly higher than that derived for AC Her from low-resolution profiles by Bujarrabal et al. (2013a), $8.4 \times 10^{-4} M_{\odot}$. This discrepancy mostly comes from the longer distance used here (the derived mass value varies approximately with $D^{2}$ ); but we recall the percentage of flux resolved out in our interferometric observations and that, as our data show, the temperature is lower than assumed in our previous work. It is difficult to estimate the effects on the derived mass value from this optically thick line because of the missing flux, but we can expect a slight underestimate of the mass. We conclude that for $D=1.6 \mathrm{kpc}$, the circumstellar mass of the detected nebula around $\mathrm{AC}$ Her must be in the range $10^{-3} M_{\odot} \lesssim M_{\text {neb }} \lesssim 2 \times 10^{-3} M_{\odot}$. We note that recent studies of the IR emission of AC Her (Hillen et al. 2015) suggest a very compact inner region, within 200 AU from the center and very thin, which contains a high dust mass, $\sim 2.5 \times 10^{-3} M_{\odot}$ (with perhaps a low gas-to-dust ratio). Our analysis can neither confirm nor rule out this component, which would be unresolved in the maps and very optically thick in ${ }^{12} \mathrm{CO} J=2-1$ (and given the uncertainties in the dust disk properties and in our modeling, particularly in the temperature of the central regions). The physical conditions derived here are just representative of the extended disk probed in our maps, while the IR data identify a very compact dust structure. We recall that AC Her has not been mapped in the ${ }^{13} \mathrm{CO}$ (less opaque) lines, hampering a proper analysis of the nebular mass from model fitting.

\section{Conclusions}

1. We presented PdBI maps of ${ }^{12} \mathrm{CO} J=2-1$ emission from the nebula surrounding the post-AGB star AC Her, in which we convincingly identified a Keplerian disk (Sects. 2 and 3). Orbiting disks are suspected to play a fundamental role in the evolution of AGB and post-AGB objects (Sect. 1), but before this work, such a structure had been clearly detected and studied in only one source (the Red Rectangle).

2. AC Her and the Red Rectangle belong to a relatively wide class of post-AGB stars in which rotating disks are suspected to exist and to represent most of the nebular mass (Sect. 1).
These are double stars whose surrounding nebulae systematically show peculiar CO line profiles (in observations with low angular resolution), similar to those expected from disks in rotation. Our maps support that Keplerian disks are systematically present in these sources and that they are, therefore, relatively common in nebulae around binary evolved stars.

3. We derived the main properties of the disk from directly interpreting the data and model fitting. We find a total disk radius of $\sim 1.7 \times 10^{16} \mathrm{~cm}$. Densities of $10^{6}-10^{4} \mathrm{~cm}^{-3}$ and temperatures of $80-20 \mathrm{~K}$ are obtained. The velocity field is found to be basically Keplerian; we do not find clear evidence of gas in expansion (as found in other similar objects).

4. We find a disk mass $\sim 1.5 \times 10^{-3} M_{\odot}$ and a central mass $\sim 1.5 M_{\odot}$, after accounting for possible underestimations due to uncertainties in the modeling (Sect. 3.1).

5. Significant differences between disks surrounding stars of this class are found. The disk in AC Her is much smaller and contains less mass than that of the Red Rectangle. It is also significantly cooler, even though the gas is closer to the center. Our data do not show any sign of outflowing gas, which is conspicuous in the Red Rectangle and dominating in the nebula around the similar object 89 Her (and, of course, in most nebulae around AGB and post-AGB stars, see Sect. 1).

6. However, the existing data on AC Her are still meager, therefore our modeling is uncertain. A fraction of the total flux is lost in the interferometric maps, which may come from an extended outflow, and the resolution is poor compared with the disk size. On the other hand, the lack of maps of optically thin lines prevents a deeper analysis of the total disk mass. We hope that future mapping of ${ }^{12} \mathrm{CO}$ and ${ }^{13} \mathrm{CO}$ lines will allow more accurate studies.

Acknowledgements. This work is based on observations carried out with the IRAM Plateau de Bure Interferometer; IRAM is supported by INSU/CNRS (France), MPG (Germany) and IGN (Spain).

\section{References}

Alcolea, J., Bujarrabal, V., Sánchez Contreras, C., Neri, R., \& Zweigle, J. 2001, A\&A, 373, 932

Alcolea, J., Neri, R., \& Bujarrabal, V. 2007, A\&A, 468, L41

Blackman, E. G., \& Lucchini, S. 2014, MNRAS, 440, L16

Bujarrabal, V., \& Alcolea, J. 2013, A\&A, 552, A116

Bujarrabal, V., Castro-Carrizo, A., Alcolea, J., \& Sánchez Contreras, C. 2001, A\&A, 377, 868

Bujarrabal, V., Castro-Carrizo, A., Alcolea, J., \& Neri, R. 2005, A\&A, 441, 1031

Bujarrabal, V., van Winckel, H., Neri, R., et al. 2007, A\&A, 468, L45

Bujarrabal, V., Young, K., \& Fong, D. 2008, A\&A, 483, 839

Bujarrabal, V., Alcolea, J., Van Winckel, H., Santander-García, M., \& CastroCarrizo, A. 2013a, A\&A, 557, A104

Bujarrabal, V., Alcolea, J., Van Winckel, H., et al. 2013b, A\&A, 557, L11

Castro-Carrizo, A., Bujarrabal, V., Sánchez Contreras, C., Alcolea, J., \& Neri, R. 2002, A\&A, 386, 633

Castro-Carrizo, A., Quintana-Lacaci, G., Neri, R., et al. 2010, A\&A, 523, A59

Castro-Carrizo, A., Neri, R., Bujarrabal, V., et al. 2012, A\&A, 545, A1

Gielen, C., Bouwman, J., van Winckel, H., et al. 2011, A\&A, 533, A99

Guilloteau, S., \& Dutrey, A. 1998, A\&A, 339, 467

Guilloteau, S., Di Folco, E., Dutrey, A., et al. 2013, A\&A, 549, A92

Hillen, M., de Vries, B. L., Menu, J., et al. 2015, A\&A, submitted

Isella, A., Testi, L., Natta, A., et al. 2007, A\&A, 469, 213

Libert, Y., Winters, J. M., Le Bertre, T., Gérard, E., \& Matthews, L. D. 2010, A\&A, 515, A112

Soker, N. 2002, ApJ, 568, 726

van Aarle, E., Van Winckel, H., Lloyd Evans, T., et al. 2011, A\&A, 530, A90

Van Winckel, H. 2003, ARA\&A, 41, 391

Van Winckel, H., Waelkens, C., Waters, L. B. F. M., et al. 1998, A\&A, 336, L17

Van Winckel, H., Lloyd Evans, T., Briquet, M., et al. 2009, A\&A, 505, 1221 